\title{
Lung ultrasound in the diagnosis of pediatric pneumonia: Are we ready for routine use?
}

\author{
Goran Roić \\ Children's Hospital Zagreb, Department \\ of Pediatric Radiology, Zagreb, Croatia \\ ${ }^{*}$ Corresponding author: \\ goran.roic@kdb.hr \\ Tel.: + 38514600274 \\ Fax.: + 38514600113
}

Received/Accepted: 25 April 2016

Key words: Children - Pneumonia - Lung " Ultrasonography.

The interest in lung ultrasound (LUS) for the use in the diagnosis and follow-up of pediatric pneumonia increased during the last few years. However, it still has not been widely excepted routine imaging tool in every day clinical practice as it should be in my opinion.

Pediatric pneumonia is the leading cause of illness and death in children worldwide and still remains a diagnostic challenge (1). Clinical signs and symptoms of pneumonia vary depending on a child's age and the etiology of infection. Presenting signs and symptoms have poor diagnostic specificity, which may further complicate the diagnosis. Traditionally, the diagnosis of pneumonia is mainly based on physical examination including auscultation, chest radiograph and laboratory evaluation. Within the physical examination, the stethoscope is broadly ac- cepted diagnostic tool in every day clinical practice (2).

Although chest radiography has been widely used for the diagnosis of pneumonia because of its convenience and ease of access, some studies have shown substantial variability in the interpretation of chest radiographs. On the other hand, the fact is that a negative chest radiograph does not eliminate the possibility of having pneumonia. Thoracic CT scan is considered the "gold standard" for detection of pneumonia and other pulmonary lesions in adults, but due to high radiation dose it cannot be used as a first-line radiological examination in pediatric patients with suspected pneumonia (3).

The lung is not an ideal target for ultrasonography, but once fluid or solid material has accumulated in the lung it can more easily be visualized on an ultrasound scan. That is the reason that lung ultrasonography is being increasingly utilized in the diagnosis of pediatric pneumonia. Furthermore, advances in ultrasound technology have made lung ultrasound an attractive, safe, portable and inexpensive option for the diagnosis of pneumonia (4). To identify pneumonia by LUS, a consolidation needs to reach the pleura and be within an intercostal window. In children, the former is offset by the relative small lung size, and may explain the relative high sensitivity. The characteristic so- 
nographic sign of lung consolidation is a sub pleural, echo-poor region with air or fluid bronchograms, comet-tail reverberation artifacts at the far-field margin, and a vascular pattern within the consolidation $(2,5,6)$. It is also useful in following up the progress of pneumonia. Multiple studies have shown LUS is sensitive diagnostic imaging tool to identify pneumonia in children, more accurate in comparison with conventional chest radiography. LUS is suggested as complementary tool to chest radiography in the diagnosis of pneumonia in children and that the follow up of pneumonia, which can reduce the exposure of children to ionizing radiation. According to a meta-analysis, LUS had high specificity and sensitivity in the hands of trained clinicians (7). As with any other ultrasound applications, LUS is operator-dependent and correct diagnosis cannot be made if the operator is insufficiently trained or inexperienced. Fortunately, it appears that the learning curve for LUS is easier and faster than for other ultrasound applications. Recommendations to train general pediatricians on LUS for the diagnosis of childhood pneumonia could have an important impact in different clinical settings, especially in limited resources countries and small primary care clinics where radiography may not be commonly available (7). Furthermore, it is very important that its application might to a certain extent exclude the need for imaging modalities based on ionizing radiation, strongly supporting the Image Gently campaign (8).

In Conclusion at the current stage, LUS is a complementary tool to chest radiography and physical examination in the diagnosis and the follow-up of pneumonia in children, allowing reduction of exposure to ionizing radiation, which is exceptionally impor- tant. Lung ultrasound is a reliable, safe, inexpensive and portable diagnostic tool, and should be implemented in everyday clinical practice whenever physical findings need to be complemented with imaging findings.

Conflict of interest: The author declares that he has no conflict of interest.

\section{References}

1. Liu L, Oza S, Hogan D, Perin J, Rudan I, Lawn JE, et al. Global, regional, and national causes of child mortality in 2000-13, with projections to inform post-2015 priorities: an updated systematic analysis. Lancet. 2015; 31;385(9966):430-40.

2. World Health Organization. Pneumonia. Fact Sheet No. 331. Available at: www. who.int/mediacentre/factsheets/fs331/en/. 2014.

3. Lovrenski J, Petrović S, Balj-Barbir S, Jokić R, Vilotijević-Dautović G. Stethoscope vs. ultrasound probe - which is more reliable in children with suspected pneumonia? Acta Med Acad. 2016;45(1):39-50.

4. Ye X, Xiao H, Chen B, Zhang SY. Accuracy of Lung Ultrasonography versus Chest Radiography for the Diagnosis of Adult Community Acquired Pneumonia: Review of the Literature and MetaAnalysis. PLoS One. 2015;10(6):e0130066.

5. Blaivas M. Lung Ultrasound in Evaluation of Pneumonia. J Ultrasound Med. 2012;31(6):823-6.

6. Caiulo VA, Gargani L, Caiulo S, Fisicaro A, Moramarco F, Latini G, et al. Lung ultrasound characteristics of community-acquired pneumonia in hospitalized children. Pediatr Pulmonol. 2013;48(3):280-7.

7. Copetti R, Cattarossi L. Ultrasound diagnosis of pneumonia in children. Radiol Med. 2008;113(2):190-8.

8. Pereda MA, Chavez MA, Hooper-Miele CC, Gilman RH, Steinhoff MC, Ellington LE, et al. Lung ultrasound for the diagnosis of pneumonia in children: a meta-analysis. Pediatrics. 2015;135(4):71422.

9. Ho MC, Ker CR, Hsu JH, Wu JR, Dai ZK, Chen IC. Usefulness of lung ultrasound in the diagnosis of community-acquired pneumonia in children. Pediatr Neonatol. 2015;56(1):40-5. 